\title{
Tumor neuroendocrino relacionado con enfermedad inflamatoria intestinal: a propósito de dos casos
}

\author{
Neuroendocrine tumor associate with inflammatory bowel disease: two cases report
}

Jeancarlos J. Trujillo-Díaz ${ }^{1 *}$, Francisco Blanco-Antona', Javier Ortiz de Solórzano-Aurusa', Gerardo Martínez-García², Luis Fernández-Salazar ${ }^{3}$ y Juan P. Beltrán-de Heredia y Rentería ${ }^{1}$

${ }^{1}$ Servicio de Cirugía General y del Aparato Digestivo; ${ }^{2}$ Servicio de Anatomía Patológica; ${ }^{3}$ Servicio del Aparato Digestivo. Hospital Clínico Universitario de Valladolid, Valladolid, España

\begin{abstract}
Resumen
Introducción: Los pacientes con enfermedad inflamatoria intestinal (Ell) tienen mayor riesgo de desarrollar neoplasias gastrointestinales y el adenocarcinoma es el relacionado con más frecuencia y el tumor neuroendocrino (TNE) el más raro. Caso clínico: Se presentan los casos de dos pacientes, uno con enfermedad de Crohn y otro con colitis ulcerosa, que cursan con clínica inespecífica, y tras resección de la lesión intestinal se diagnostica un TNE gastrointestinal (TNEGI). Discusión y conclusiones: Los TNEGI tienen una clínica insidiosa y pueden confundirse con los de la Ell. Es posible un nexo entre ambas entidades, lo que sugiere un papel importante del proceso inflamatorio crónico intestinal. El tratamiento de elección de los TNEGI es la resección.
\end{abstract}

PALABRAS CLAVE: Enfermedad inflamatoria intestinal. Tumor neuroendocrino. Enfermedad de Crohn. Colitis ulcerosa. Tumor neuroendocrino intestinal.

\begin{abstract}
Introduction: Patients with inflammatory bowel disease (IBD) have a higher risk of developing gastrointestinal tumors, the adenocarcinoma is the most frequently associated, and neuroendocrine tumor (NET) the most rare. Clinical cases: We present two patients, one with Crohn's disease and the other with ulcerative colitis, who present nonspecific symptoms, and after resection of an intestinal lesion, a gastrointestinal NET (GINET) is diagnosed. Discussion and conclusion: The GINET have an insidious clinic and these can be confused with those of the IBD. There could be an association between both pathologies; an important role of the chronic intestinal inflammatory process is suggested. The best treatment for GINET is the resection.
\end{abstract}

KEY WORDS: Inflammatory bowel disease. Neuroendocrine tumor. Crohn's disease. Ulcerative colitis. Neuroendocrine bowel tumor.

\section{Introducción}

Los pacientes con enfermedad inflamatoria intestinal (EII), sobre todo aquéllos con enfermedad de Crohn (EC), muestran mayor riesgo de sufrir neoplasias gastrointestinales; se calcula que cerca de $2 \%$ las desarrollaran durante el curso de la afección, en particular los individuos bajo tratamiento inmunosupresor o que lo han recibido".

\footnotetext{
Correspondencia:

*Jeancarlos Jhosmer Trujillo-Díaz

Avda. Ramón y Cajal, 3

C.P. 47003 , Valladolid, España

E-mail: jeancar5671@gmail.com
}

Cir Cir. 2019;87(S1):17-21

Contents available at PubMed www.cirugiaycirujanos.com 
La mayor parte de las neoplasias vinculadas con la Ell aparece en los segmentos intestinales inflamados y sigue la secuencia inflamación-displasia-carcinoma. Dentro de los tumores gastrointestinales observados en la Ell, el más frecuente es el adenocarcinoma y su nexo con un tumor neuroendocrino (TNE) es un fenómeno menos común².

Los TNE gastrointestinales (TNEGI) tienen casi siempre una presentación clínica insidiosa, con síntomas poco específicos que pueden confundirse con los de la Ell con facilidad por lo que su diagnóstico se establece por lo general tras su resección endoscópica o quirúrgica ${ }^{3}$.

Se presentan los casos de dos pacientes con Ell, en los que se diagnosticó un TNEGI, y se realiza una revisión de los aspectos clínicos, diagnósticos y terapéuticos de esta rara relación.

\section{Casos clínicos}

\section{Primer paciente}

Varón de 74 años con antecedentes personales de hipertensión arterial, hiperplasia prostática benigna y carcinoma renal de células claras tratado con tumorectomía renal. Padece EC, con afectación del íleon terminal, de larga evolución (bajo seguimiento por el Servicio Digestivo desde seis años antes) con cuadros de oclusión intestinal repetidos que se resuelven con medidas conservadoras. En tratamiento actual incluye mesalazina y había recibido con anterioridad tratamiento con azatioprina (durante cinco meses seis años antes). Ingresa por nuevo episodio de oclusión intestinal. Se solicita tomografía computarizada (TC) que delinea un engrosamiento mural de un segmento de íleon terminal de $6 \mathrm{~cm}$ de longitud que causa dilatación retrógrada (Fig. 1A). En la enterorresonancia magnética (RM) se visualiza una estenosis segmentaria del íleon terminal debido a un engrosamiento parietal, en un segmento, a unos $5 \mathrm{~cm}$ de la válvula ileocecal (Fig. 1B). Ante la falta de mejoría del cuadro clínico, con el diagnóstico de EC ileal complicada y resistente al tratamiento médico, se decide intervenir de manera quirúrgica. Se practicó un abordaje laparoscópico con cuatro trócares que identifica una zona de estenosis en el íleon terminal $(\sim 10 \mathrm{~cm}$ de extensión) con paredes engrosadas y adenopatías aumentadas de tamaño (Fig. 2A). Se efectúa una resección ileocecal, con anastomosis manual isoperistáltica intracorpórea. El postoperatorio cursa de modo favorable sin complicaciones y el sujeto recibe el alta médica al quinto día postoperatorio. El estudio anatomopatológico de la pieza quirúrgica revela una Ell tipo EC con intensa actividad inflamatoria y en la zona de estenosis tienen un tumor infiltrante casi submucoso en su totalidad de $2.7 \mathrm{~cm}$ de diámetro que infiltra la pared intestinal y alcanza la grasa mesentérica, con invasión vascular y linfática de forma extensa (Figs. 2B y C). La actividad mitótica era $<2$ mitosis y el Ki-67 < $2 \%$ por 10 CGA. En el estudio inmunohistoquímico, las células neoplásicas expresaban positividad para los marcadores cromogranina A, sinaptofisina, CD56 y somatostatina focalmente (Fig. 2D). Los marcadores para insulina, glucagón, gastrina y VIP fueron negativos. El tumor metastatizaba a cinco de 17 ganglios linfáticos. Se estableció el diagnóstico de tumor neuroendocrino grado G1, estadio IIIb (T4N1M0)4. En la revisión a los dos meses del alta se encontraba asintomático y la determinación de $\mathrm{CgA}$ en sangre y una gammagrafía con octeótrido fueron normales.

\section{Segundo paciente}

Varón de 52 años con antecedentes personales de fumador y pólipos hiperplásicos en el colon. Sufre CU de larga evolución (24 años) en tratamiento con mesalazina, aunque ha recibido antes tratamiento con azatioprina (un ciclo 22 años antes). En una colonoscopia de control se visualiza una tumoración redondeada submucosa de 5-7 $\mathrm{mm}$ en el recto distal con mucosa normal (Fig. 3A). Se practica una ecoendoscopia y se identifica una lesión homogénea unos 6 a $8 \mathrm{~cm}$ del margen anal, bien delimitada y circunscrita a la submucosa de 6 × 9 mm (Fig. 3B). Se lleva a cabo resección endoscópica de la tumoración con capuchón y banda elástica (Fig. 3C-D). El informe anatomopatológico reveló un tumor de $0.9 \mathrm{~cm}$ de diámetro localizado en el estrato muscular sin invasión linfovascular y con bordes de resección libres. El estudio inmunohistoquímico fue positivo para CgA y sinaptofisina. El índice Ki67 era < 1\%. El diagnóstico definitivo fue tumor neuroendocrino G1, estadio I (T1NOM0) ${ }^{4}$. Con el diagnóstico de TNE rectal se solicitan TC abdominopélvica, gammagrafía con ${ }^{111}$ In-octeótrido completada con adquisición de SPECT-CT y una determinación de CgA en sangre que fueron normales. Luego de dos años y medio de seguimiento se encuentra asintomático con controles analíticos y endoscópicos sin signos de recidiva tumoral.

\section{Discusión}

Existe evidencia acerca de la relación entre pacientes con Ell y TNE. West publicó una serie con una 

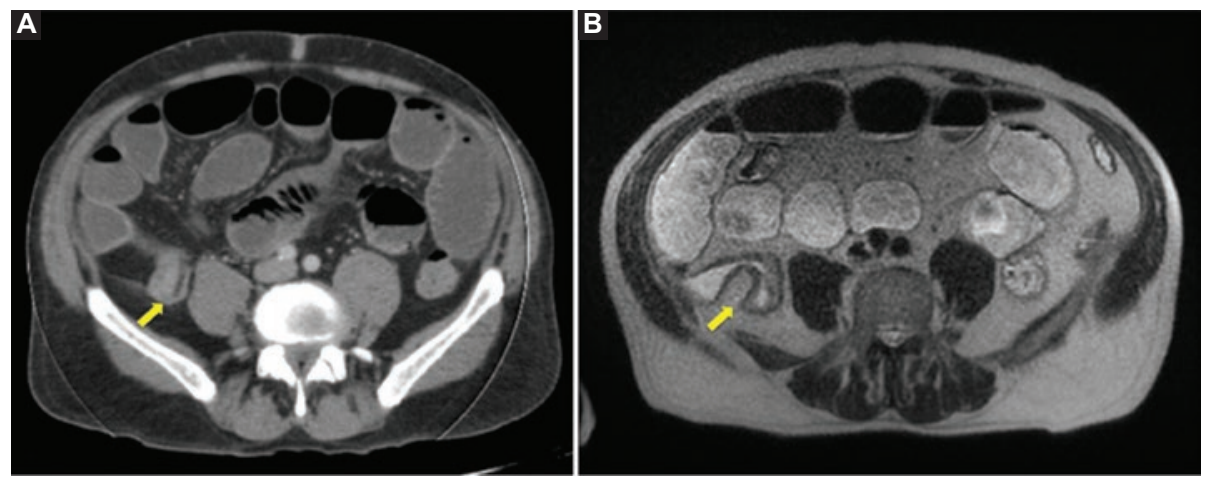

Figura 1. A: TC que revela engrosamiento mural de un segmento del íleon terminal (flecha); B: RM que muestra el mismo engrosamiento parietal en relación con estenosis (flecha).

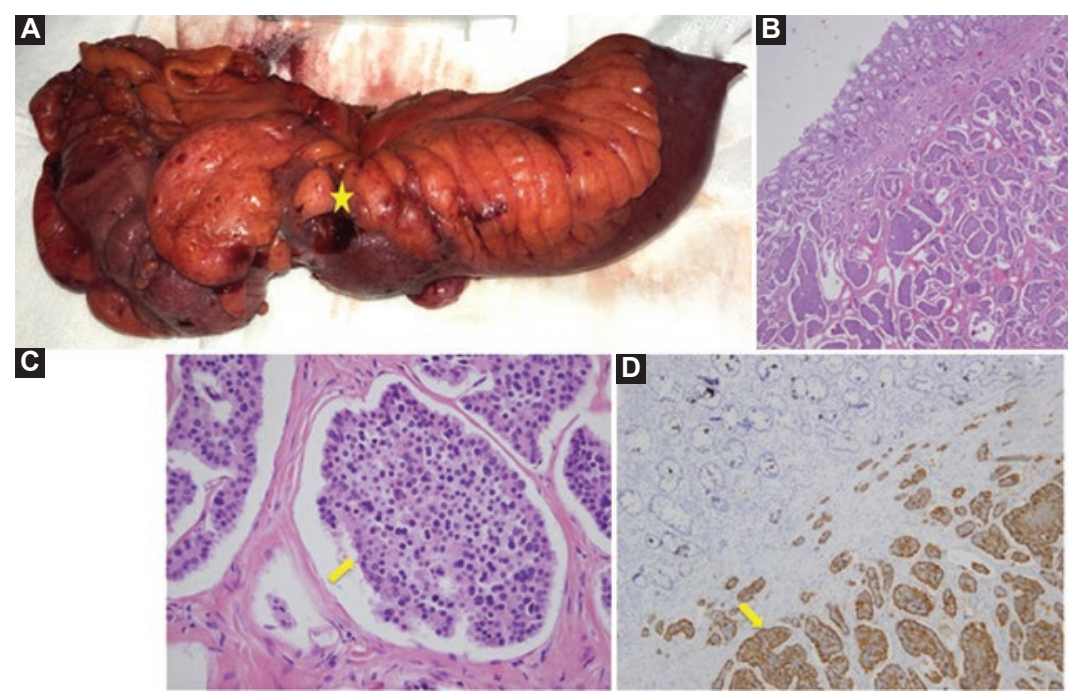

Figura2. Cirugía:A: Piezaquirúrgica(ciegoe íleonterminal)enlaquese visualizaunazonadeestenosisporlatumoración( $\star$ ); B: Histología(H-E4X)se reconoce un patrón organoide separado por tabiques finos; C: Histología (H-E 40X) monomorfismo celular con cromatina fina y granular con patrón de "sal y pimienta" (flecha); D: Inmunohistoquímica: tinción citoplásmica y granular positiva a cromogranina (flecha).
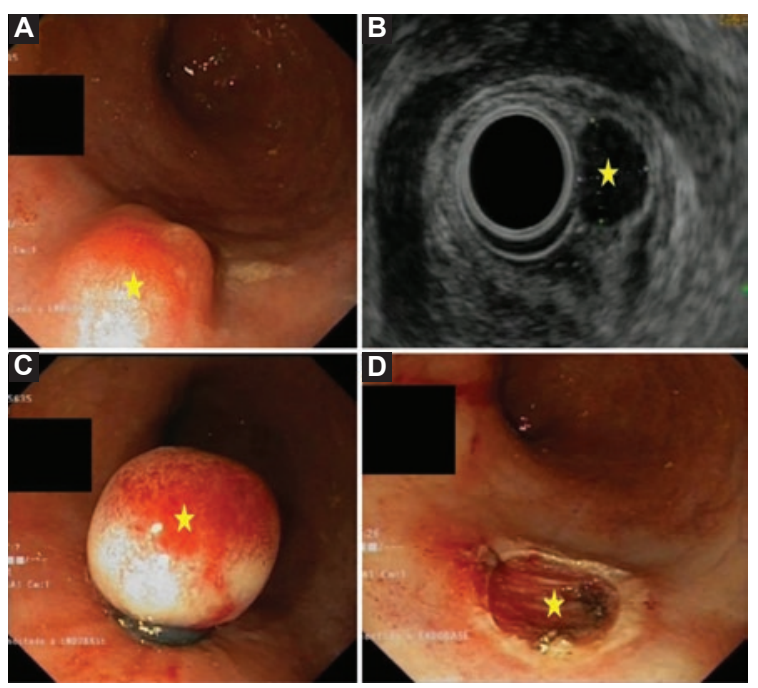

Figura 3. A: Colonoscopia: tumoración submucosa redondeada $(\star)$; B: Ecoendoscopia: lesión homogénea, submucosa y milimétrica ( $\star$ ); C: Resección endoscópica de la tumoración con capuchón y banda elástica ( $\star$ ); D: Base de resección libre de infiltración ( $\star$ ). incidencia de TNE 14.9 veces mayor en pacientes con EC que en el grupo control ${ }^{5}$. Hasta la actualidad se han descrito 58 pacientes con un TNE y una Ell. Casi todos son hallazgos incidentales en el curso de la operación realizada para el tratamiento de la Ell2. Cuando coexisten estas dos anomalías, el TNE tiende a ser más agresivo y tener un peor pronóstico ${ }^{6}$.

La teoría más aceptada para explicar el nexo entre TNEGI y Ell sugiere un papel importante del proceso inflamatorio crónico intestinal, en el que las citocinas proinflamatorias, como TNF, IFN $\gamma$, IL-2, causan una hiperestimulación e hiperplasia de las células enteroendocrinas ${ }^{7,8}$. Estas citocinas pueden actuar a distancia, lo que explica la aparición de TNEGI en segmentos no afectados por la Ell9.

Los TNE gastrointestinales (TNEGI) provienen de las células enterocromafines de las criptas intestinales, son las neoplasias malignas más comunes del 
intestino delgado distal y su localización más frecuente es el intestino delgado (45\%), seguido del recto (20\%). Por lo regular, los TNE situados en el intestino delgado se encuentran en el íleon terminal ${ }^{2,10}$.

La tasa de incidencia de los TNE ha aumentado en los últimos años; en la actualidad es de 5.25 por cada 100000 habitantes/año. No existe diferencia en la incidencia entre hombres y mujeres y la media de edad de presentación es de 66 años ${ }^{11}$.

Los TNEGI son casi siempre asintomáticos hasta etapas avanzadas de la enfermedad, por lo que pueden tener una progresión local en el momento de la presentación clínica o del diagnóstico ${ }^{12}$. Cuando son sintomáticos, pueden simular un brote de $\mathrm{EII}^{13}$, lo que dificulta aún más su diagnóstico; Hsu informó que 2.3\% de los pacientes con tumor carcinoide ileal se diagnosticó y trató de manera inicial como $\mathrm{EC}^{14}$. Es habitual que se encuentren de forma incidental en intervenciones quirúrgicas o en exploraciones endoscópicas en campañas de cribado o por enfermedad digestiva, como sucedió en el caso de estos pacientes.

Los TNEGI pueden presentarse en clínica de forma aguda como cuadros de oclusión intestinal ( $90 \%$ de los casos) o como episodios de hemorragia gastrointestinal. Dentro de las manifestaciones crónicas figuran el dolor abdominal, que suele diagnosticarse como síndrome de malabsorción o de colon irritable, y la pérdida de peso $^{3}$. El síndrome carcinoide puede aparecer cuando existen metástasis hepáticas y es raro en los TNE rectales.

Para el diagnóstico bioquímico de los TNEGI se emplea la cromogranina A como marcador y la determinación de 5-HIAA en orina, que muchas veces está alterada en casos de enfermedad avanzada?.

Las características radiológicas de los TNEGI pueden ser similares a las de la Ell, en especial la EC, en cuanto a la rigidez y las irregularidades de la pared intestinal ${ }^{12}$. El estudio de elección inicial en casos de sospecha de TNEGI intestinales es la TC, que tiene una sensibilidad del $73 \%$, y sirve para detectar el tumor primario y las metástasis, y planear el tratamiento quirúrgico. La resonancia magnética es el mejor estudio para definir las metástasis hepáticas con una sensibilidad de $95 \%{ }^{15}$.

La gammagrafía con análogos de la somatostatina (Octreoscan) sirve para el diagnóstico y para predecir la respuesta al tratamiento médico, y posee una sensibilidad de $83 \%$ y un valor predictivo positivo de $100 \%$, aunque se han registrado casos en que la EC puede causar falsos positivos ${ }^{2,16}$.
El tratamiento de elección de los TNEGI es la resección. En los tumores del intestino delgado se debe realizar una exéresis quirúrgica amplia que incluya mesenterio y ganglios linfáticos regionales. En Ios TNE rectales, el tratamiento depende de su tamaño o factores de riesgo (infiltración de la muscular propia, invasión linfovascular, un índice mitótico > 2/10 HPF o un Ki-67 > 2\%) y varía desde la resección endoscópica en los tumores $<1 \mathrm{~cm}$ hasta la resección de recto en las malformaciones $>2 \mathrm{~cm}^{17,18}$.

En presencia de invasión retroperitoneal o grandes vasos, la resección quirúrgica citorreductora paliativa puede ser adecuada con el fin de disminuir los síntomas sistémicos y prevenir las complicaciones locales. En la enfermedad metastásica hepática, la resección abarca desde una metastasectomía hasta un trasplante hepático. Como tratamientos alternativos o adjuntos pueden emplearse la ablación con radiofrecuencia, la crioterapia y la quimioembolización transarterial ${ }^{2,19}$.

En el tratamiento sistémico de los TNEGI metastásicos, localmente avanzados o G3 con mal pronóstico se administran los ASS, el interferón y diversos regímenes de quimioterapia convencional ${ }^{2,20}$. La radioterapia está indicada en las metástasis óseas o del sistema nervioso central ${ }^{3}$.

\section{Conclusión}

Como conclusión puede señalarse que, debido a la relación entre los TNEGI y la Ell, y dado que la clínica de las dos entidades puede ser similar, hay que sospechar la presencia de estos tumores en pacientes con Ell de larga evolución, que cursen con síntomas inespecíficos y que recibieron tratamiento inmunosupresor, como ocurrió en los casos presentados.

\section{Conflicto de intereses}

Los autores declaran que no tienen conflictos de interés.

\section{Financiamiento}

Este trabajo no ha requerido financiamiento.

\section{Responsabilidades éticas}

Protección de personas y animales. Los autores declaran que para esta investigación no se han realizado experimentos en seres humanos ni en animales. 
Confidencialidad de los datos. Los autores declaran que han seguido los protocolos de su centro de trabajo sobre la publicación de datos de pacientes.

Derecho a la privacidad y consentimiento informado. Los autores han obtenido el consentimiento informado de los pacientes y/o sujetos referidos en el artículo. Este documento obra en poder del autor de correspondencia.

\section{Bibliografía}

1. Cioffi U, De Simone M, Ferrero S, Ciulla M, Lemos A, Avesani E. Synchronous adenocarcinoma and carcinoid tumor of the terminal ileum in a Crohn's disease patient. BMC Cancer. 20058;5:157.

2. Pellino G, Marcellinaro R, Candilio G, De Fatico G, Guadagno E, Campione et al. The experience of a referral centre and literature overview of GIST and carcinoid tumours in inflammatory bowel diseases. Int J Surg. 2016;28(Suppl 1):S133-41. doi: 10.1016/j.jisu.2015.12.051. Epub 2015 Dec 18.

3. Mussan-Chelminsky G, Vidal-González P, Núñez-García E, Valencia-García L, Márquez-Ugalde M. Intestinal carcinoid tumour: case report. Cir Cir. 2015;83(5):438-41. doi: 10.1016/j.circir.2015.05.047. Epub 2015 Aug 28.

4. Brierley J, Gospodarowicz M, Wittekind C (eds.). TNM Classification of malignant tumours. $8^{\text {th }}$ edition. Wiley-Blackwell, 2017.

5. West N, Wise P, Herline A, Muldoon R, Chopp W, Schwartz D. Carcinoid tumors are 15 times more common in patients with Crohn's disease. Inflamm Bowel Dis. 2007;13(9):1129-34.

6. Bassi A, Loughran C, Foster P. Carcinoid tumour of the terminal ileum simulating Crohn disease. Scand J Gastroenterol. 2003;38(9):1004-6.

7. Boltin D, Levi Z, Halpern M, Fraser G. Concurrent small bowel adenocarcinoma and carcinoid tumor in Crohn's disease-case report and literature review. J Crohns Colitis. 2011;5(5):461-4. doi: 10.1016/j.crohns.2011.04.006. Epub 2011 May 10.

8. Mogollón M, Conde R, Segura I, Huertas F, San Miguel C, Ferrón J, et al. Ileal carcinoid tumor within Crohn's disease. Rev Esp Enferm Dig. 2014;106(4):298-9.

9. Samlani-Sebbane Z, Gharaba S, Narjis Y, Rabbani K, Diffaa A, Krati K, et al. The coexistence of carcinoid tumours and ileal Crohn's disease: a new case. Acta Endosc 2012:42:194. doi: 10.1007/s10190-012-0265-1.

10. Komborozos V. Carcinoid tumours of the small bowel colon andrectum. Hellenic J Surgery. 2011;83:12-20. doi: 10.1007/s13126-011-0003-8.

11. Moris D, Ntanasis-Stathopoulos I, Tsilimigras D, Vagios S, Karamitros A, Karaolanis G, et al. Update on surgical management of small bowel neuroendocrine tumors. Anticancer Res. 2018;38(3):1267-1278.

12. Bakker C, Sosef M, Vliegen R. A wolf in sheep's clothing. BMJ Case Rep. 201125;2011. pii: bcr1120103492. doi: 10.1136/bcr.11.2010.3492.

13. Praticò C, Rizzello F, Fornarini G, Calafiore A, Calabrese C, Campieri M, et al. Four cases of carcinoid tumour in Crohn's disease: coincidence or correlation? Int J Colorectal Dis. 2013;28(12):1743-5. doi: 10.1007/ s00384-013-1732-7. Epub 2013 Jun 15.

14. Hsu E, Feldman J, Lichtenstein G. Ileal carcinoid tumors stimulating Crohn's disease: incidence among 176 consecutive cases of ileal carcinoid. Am J Gastroenterol. 1997;92(11):2062-5.

15. Maxwell J, Howe J. Imaging in neuroendocrine tumors: an update for the clinician. Int J Endocr Oncol. 2015; 2(2):159-168. doi:10.2217/ije.14.40.

16. Fernández A, Tabuenca O, Peteiro A. A "false positive" octreoscan in ileal Crohn's disease. World J Gastroenterol. 2008;14(34):5349-52.

17. McDermott F, Heeney A, Courtney D, Mohan H, Winter D. Rectal carcinoids: a systematic review. Surg Endosc. 2014;28(7):2020-6. doi: 10.1007/ s00464-014-3430-0. Epub 2014 Mar 1.

18. Ramage J, Ahmed A, Ardill J, Bax N, Breen D, Caplin M, et al. Guidelines for the management of gastroenteropancreatic neuroendocrine (including carcinoid) tumours (NETs) Gut 2012;61:6-32. doi: 10.1136/gutjnl-2011-300831. Epub 2011 Nov 3.

19. Mandair D, Caplin M. Colonic and rectal NET's. Best Pract Res Clin Gastroenterol. 2012;26(6):775-89. doi: 10.1016/j.bpg.2013.01.007.

20. Sorbye H, Strosberg J, Baudin E, Klimstra D, Yao J. Gastroenteropancreatic high-grade neuroendocrine carcinoma. Cancer. 2014;120(18):2814-23. doi: 10.1002/cncr.28721. Epub 2014 Apr 25. 